\title{
Biospecimen Type
}

National Cancer Institute

\section{Source}

National Cancer Institute. Biospecimen Type. NCI Thesaurus. Code C70713.

The type of a material sample taken from a biological entity for testing, diagnostic,

propagation, treatment or research purposes. This includes particular types of cellular molecules, cells, tissues, organs, body fluids, embryos, and body excretory substances. 